\title{
Laser Palatoplasty versus Laser Palatoplasty and Laser Turbinectomy for Snoring and Mild Obstructive Sleep Apnea
}

\author{
Nasser Naguib Nasr, Sherif Mohammad Askar, Loay Mohamed Farid*, Ali Awad \\ Department of Otorhinolaryngology, Faculty of Medicine Zagazig University, Egypt. \\ *Corresponding Author: Loay Mohamed Farid, Mobile: (+20)01148696583, Email: loayamir93@gmail.com
}

\begin{abstract}
Background: Patients with obstructive sleep apnea (OSA) have both anatomic and physiologic dysfunction of the upper airway during sleep resulting in repeated airway obstruction and varying degrees of hypoxemia. In patients with obstructive sleep apnea suffering from both snoring and nasal obstruction, nasal surgery relieves snoring and improves apnea/hypopnea index (AHI), lowest $\mathrm{O}_{2}$ saturation (LOS), epworth sleepness scale (ESS) and snoring severity scale (SSS) by different proportions.

Objective: To compare AHI, SSS, and ESS in laser palatoplasty and combined laser palatoplasty and laser turbinoplasty.

Patients and Methods: All patients underwent surgery had snoring and mild OSA symptoms, aged between 20 and 60 years with continuous positive airway pressure (CPAP) refusal, failure or non-compliance, classified according to type of surgical intervention into: group A 14 patients were treated with laser palatoplasty and laser turbinoplasty. Group B: 14 patients were treated with laser palatoplasty only. Pre and postoperative sleep study, Epworth sleepiness scale, and snoring score were reported and compared.

Results: As regard AHI there was no significant difference between 2 groups at pre or post, according to ESS (epworth sleepness scale) there was no significant difference between 2 groups pre and post. Regarding SSS the preoperative snoring score was reported to be significantly more in patients who had associated nasal obstruction (group A). But after surgery, the difference in postoperative values was nonsignificant reflecting the value of performing nasal surgery in this group of patients. Conclusion: Combined nasal and palatal surgery is more effective for snoring and mild OSA than palatal surgery only.
\end{abstract}

Keywords: Mild obstructive sleep apnea, Nose, Palatal surgery.

\section{INTRODUCTION}

More than one third of all people snore regularly. Snoring is a common accompaniment of obstructive sleep apnea (OSA) and is often disruptive for the bed partner ${ }^{(\mathbf{1})}$. Laser-assisted uvulopalatoplasty (LAUP) technique constituted first choice surgical treatment ${ }^{(2)}$. However, it is only able to improve the condition in 50\% of all obstructive sleep apnea syndrome (OSAS) patients. Friedman et al. ${ }^{(3)}$ reported a $41 \%$ success rate with this technique.

Laser turbinoplasty is a simple and well-tolerated treatment for nasal obstruction. It appears to be a safe and effective adjunct surgical procedure for snorer with nasal obstruction on an outpatient basis ${ }^{(4)}$. The nasal valve is the narrowest part of the upper airway. Because the upper airway accounts for two-thirds of the entire airway that extends down to the alveoli, simple widening of the nasal valve and the entire nasal passage would significantly contribute to a decrease in negative pressure at inspiration during sleep and contribute to improve OSA ${ }^{(5)}$.

This study aimed to compare AHI, SSS, ESS (epworth sleepness scale) in laser palatoplasty and combined laser palatoplasty and laser turbinoplasty.

\section{PATIENT AND METHODS}

The present study is a comparative prospective study. It was conducted over the period from April 2020 to May 2021 in Otorhinolaryngology Head and Neck Surgery Department, Zagazig University Hospitals.
The study included twenty eight adult patients who had snoring and mild OSA symptoms, aged between 20 and 60 years with CPAP refusal, failure or noncompliance, body mass index $(\mathrm{BMI}) \leq 35$, being fit for general anesthesia, with anterior posterior retropalatal collapse, Friedman tonsillar size grade 0 and 1 with or without nasal obstruction due to inferior turbinate hypertrophy.

Patients were divided into two equal groups: Group A: was treated with laser palatoplasty and laser turbinoplasty, and Group B: was treated with laser palatoplasty.

We excluded patients with age $<20$ and $>60$ year, body mass index $(\mathrm{BMI})>35$, moderate or severe OSA, deviated nasal septum, hypopharyngeal collapse with prominent tongue base, concentric and transverse retropalatal collapse and Friedman tonsillar size grade 2,3 and 4 .

After detailed history taking flexible nasoendoscopy during Muller maneuver, polysomnography, and drug induced sleep endoscopy was performed for all patients. Moreover, all patients had subjective analysis with the Epworth Sleepiness Scale (ESS) as a measure of daytime somnolence.

Six months after surgery, all patients underwent nasopharyngolaryngoscopy as part of the standard postsurgical protocol using the Muller maneuver. A postoperative sleep study, ESS (epworth sleepness scale), snoring score were also performed and assessed. 


\section{Ethical consent:}

An approval of the study was obtained from Zagazig University Academic and Ethical Committee. Every patient signed an informed written consent for acceptance of the operation and sharing in the study. This work has been carried out in accordance with The Code of Ethics of the World Medical Association (Declaration of Helsinki) for studies involving humans.

\section{Statistical Analysis}

The collected data were coded, processed and analyzed using the SPSS (Statistical Package for the Social Sciences) version 22 for Windows ${ }^{\circledR}$ (IBM SPSS Inc, Chicago, IL, USA). Data were tested for normal distribution using the Shapiro Wilk test. Qualitative data were represented as frequencies and relative percentages. Chi square test $\left(\chi^{2}\right)$ was used to calculate difference between the two groups of qualitative variables. Quantitative data were expressed as mean \pm SD (Standard deviation). Independent samples t-test was used to compare between two independent groups of normally distributed variables (parametric data). $P$ value $<0.05$ was considered significant.

\section{RESULTS}

As regard age and sex there was no significant difference between the 2 groups and the majority were male in both groups (Table 1). Neck circumference was $40.06 \pm 2.21$ and $40.13 \pm 1.55$ respectively with no significant difference between groups.

Table (1): Age and sex comparison between studied groups

\begin{tabular}{|c|c|c|c|c|c|}
\hline & Group A & Group B & $\mathbf{P}$ \\
\hline \multicolumn{3}{|c|}{ Age } & $44.66 \pm 8.44$ & $42.66 \pm 8.03$ & 0.502 \\
\hline \multirow[t]{4}{*}{$\operatorname{Sex}$} & Female & $\mathrm{N}$ & 4 & 5 & \multirow{4}{*}{0.69} \\
\hline & & $\%$ & $28.5 \%$ & $35.7 \%$ & \\
\hline & \multirow[t]{2}{*}{ Male } & $\mathrm{N}$ & 10 & 9 & \\
\hline & & $\%$ & $71.5 \%$ & $64.3 \%$ & \\
\hline \multirow{2}{*}{\multicolumn{2}{|c|}{ Total }} & $\mathrm{N}$ & 14 & 14 & \\
\hline & & $\%$ & $100.0 \%$ & $100.0 \%$ & \\
\hline
\end{tabular}

Table 2 shows no significant difference between groups as regard Friedman tonsillar size.

Table (2): Friedman tonsillar size distribution between studied groups

\begin{tabular}{|l|c|c|c|}
\hline & Group A & Group B & P \\
\hline Grade & 6 & 7 & \\
zero & $42.8 \%$ & $50 \%$ & \multirow{2}{*}{0.71} \\
\cline { 1 - 3 } Grade I & 8 & 7 & \\
& $57.2 \%$ & $50 \%$ & \\
\hline
\end{tabular}

Table 3 shows no significant difference between groups regarding retropalatal collapse Muller endoscopy. No significant difference between groups as regard Mallampati palatal position.
Table (3): Retropalatal collapse Muller endoscopy distribution between studied groups

\begin{tabular}{|l|c|c|c|}
\hline & Group A & Group B & P \\
\hline Grade I & 2 & 2 & \\
& $14.3 \%$ & $14.3 \%$ & \\
\cline { 1 - 3 } Grade II & 4 & 3 & \\
& $28.5 \%$ & $21.4 \%$ & \multirow{2}{*}{0.83} \\
\cline { 1 - 3 } Grade III & 6 & 5 & \\
& $42.9 \%$ & $35.8 \%$ & \\
\cline { 1 - 3 } Grade IV & 2 & 4 & \\
& $14.3 \%$ & $28.5 \%$ & \\
\hline
\end{tabular}

Regarding AHI there was no significant difference between groups at pre or post but there was significant decrease in both groups between pre and post Figure 1.

Regarding ESS (epworth sleepness scale) there was no significant difference between groups at pre and post. Regarding SSS the preoperative snoring score was reported to be significantly more in patients who had associated nasal obstruction (group A) ( $\mathrm{P}^{1 / 4}$ 0.0113). But after surgery, the difference in postoperative values was nonsignificant ( $\mathrm{P} 1 / 4$ 0.1296) reflecting the value of performing nasal surgery in this group of patients.

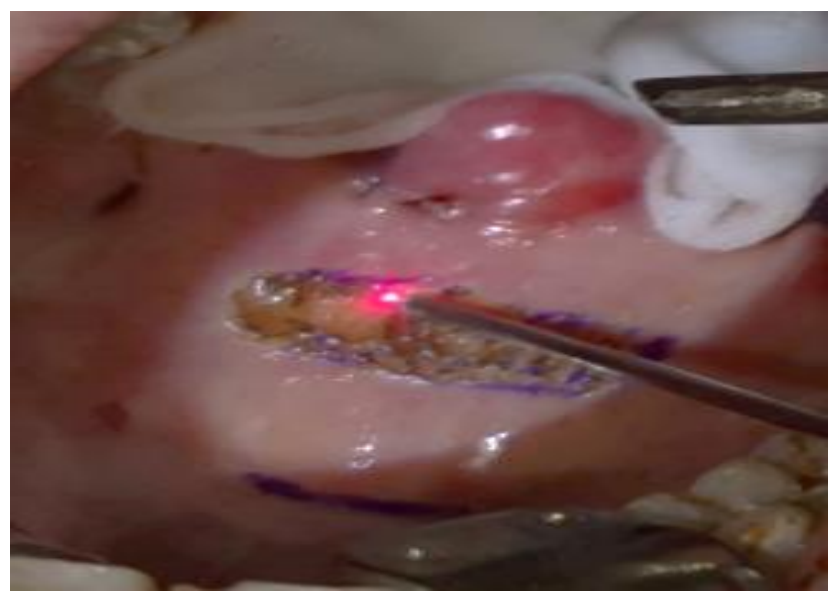

Figure (1): Intraoperative laser palatoplasty.

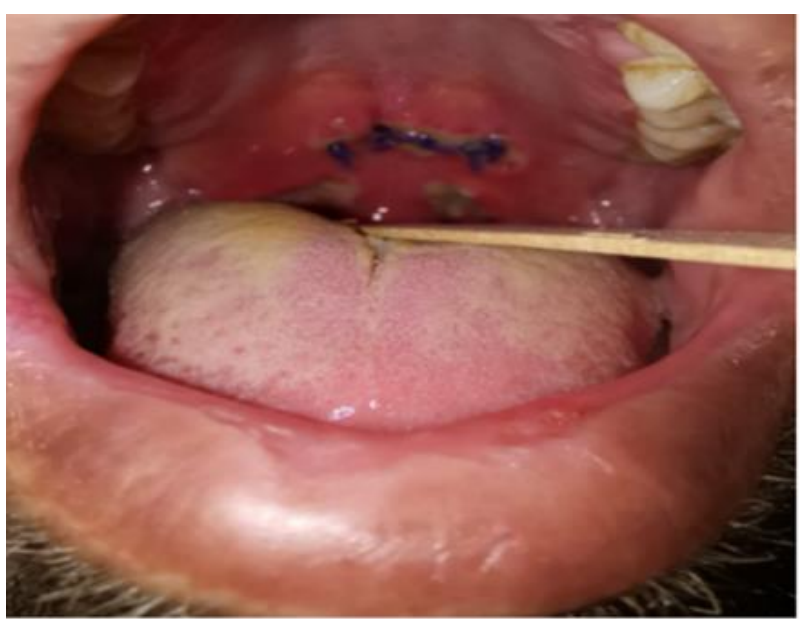

Figure (2): Palate after 1 week. 


\section{DISCUSSION}

Laser assisted uvulopalatoplasty (LAUP): is a method of treatment for snoring and obstructive sleep apnea (OSA) that was first described in 1990 by Kamami. The procedure was based on progressive widening of the oropharynx by "successive vaporizations of the vibrating soft palate, wide posterior tonsil pillars, and redundant posterior pharyngeal mucosa" to prevent obstructions during sleep ${ }^{(6)}$. Studies also showed that in adults, artificial nasal obstruction decreased the quality of sleep and increased the number of arousals, apnea, and hypopnea ${ }^{(7)}$.

In patients with obstructive sleep apnea suffering from both snoring and nasal obstruction, nasal surgery relieves snoring in $12 \%{ }^{(\boldsymbol{8})}$.

Laser-assisted turbinate surgery causes limited submucosal scarring and obliteration of the venous sinusoids, shrinking the turbinate and relieving nasal obstruction. The diode laser is the most portable and least expensive of the lasers available for rhinologic applications today ${ }^{(9)}$. Laser surgery has the advantages of limited tissue trauma and reduced bleeding. Different lasers, such as carbon dioxide $\left(\mathrm{CO}_{2}\right)$, neodymium: yttrium- aluminum garnet (Nd:YAG), holmium:yttrium-aluminum garnet (Ho:YAG), potassium titanyl phosphate (KTP), diode, and argon plasma lasers, have been used to treat ITH ${ }^{\left({ }^{(10)}\right.}$.

Our results are also consistent with the study by Friedman et al. who reported that symptoms of OSA improved after nasal surgery as regards nasal breathing, snoring, and day-time energy levels. Generally, correction of an obstructed nasal airway ameliorates symptoms in OSA patients ${ }^{(11)}$.

\section{CONCLUSION}

Combined nasal and palatal surgery is more effective for snoring and mild OSA than palatal surgery only.
Financial support and sponsorship: Nil.

\section{Conflict of Interest: Nil.}

\section{REFERENCES}

1. Janott C, Schuller B, Heiser C (2017): Acoustic information in snoring noises. HNO, 65(2):107-116.

2. Goldstein N, Post J, Rosenfeld R et al. (2000): Impact of tonsillectomy and adenoidectomy on child behavior. Arch Otolaryngol Head Neck Surg., 126:494-8.

3. Friedman M, Ibrahim H, Lee G et al. (2003): Combined uvulopalatopharyngoplasty and radiofrequency tongue base reduction for treatment of obstructive sleep apnea/hypopnea syndrome. Otolaryngol Head Neck Surg., 129:611-21.

4. Neruntarat C (2005): Laser-assisted outpatient septoplasty and laser-assisted uvulopalatoplasty for nasal obstruction and snoring. J Med Assoc Thai., 1:66-72.

5. Kohler M, Bloch K, Stradling J (2007): The role of the nose in the pathogenesis of obstructive sleep apnea and snoring. Eur Respir J., 30:1208-1215.

6. Kamami Y (1990): Laser $\mathrm{CO}_{2}$ for snoring. Preliminary results. Acta Otorhinolaryngol Belg., 44(4):451-456.

7. Hudgel D, Hendricks C (1988): Palate and hypopharynx-sites of inspiratory narrowing of the upper airway during sleep. Am Rev Respir Dis., 138:15421547.

8. Li H, Lee L, Wang P et al. (2008): Nasal surgery for snoring in patients with obstructive sleep apnea. Laryngoscope, 118(2):354-9.

9. Volk G, Pantel M, Guntinas-Lichius O et al. (2010): Prognostic value of anterior rhinomanometry in diode laser turbinoplasty. Arch Otolaryngol Head Neck Surg., 136: 1015-9.

10. Chang C, Ries W (2004): Surgical treatment of the inferior turbinate: new techniques. Curr Opin Otolaryngol Head Neck Surg., 12: 53-7.

11. Friedman M, Tanyer H, Lim J et al. (2000): Effect of improved nasal breathing on obstructive sleep apnea. Otolaryngol Head Neck Surg., 122:71-74. 